Psychotherapeut

https://doi.org/10.1007/s00278-022-00572-3

(๑) The Author(s), under exclusive licence to Springer Medizin Verlag GmbH, ein Teil von Springer Nature 2022

\section{Erratum zu: Psychotherapie - Ein kompetenzorientiertes Lehrbuch für die Zeitschrift Psychotherapeut}

Eva-Lotta Brakemeier

Universität Greifswald, Greifswald, Deutschland

\section{Erratum zu:}

Psychotherapeut 2021

https://doi.org/10.1007/s00278-021-0055

6-9

Der Titel dieses Artikels war fälschlicherweise mit "Psychotherapie - Ein kompetenzorientiertes Lehrbuch für die Zeitschrift Psychotherapeut" angegeben. Richtig wäre "Psychotherapie - Ein kompetenzorientiertes Lehrbuch" gewesen.

Der Originalbeitrag wurde korrigiert.

\section{Korrespondenzadresse}

Prof. Dr. Eva-Lotta Brakemeier

Universität Greifswald

Franz-Mehring-Str. 47, 17489 Greifswald,

Deutschland

eva-lotta.brakemeier@uni-greifswald.de
Die Online-Version des Originalartikels ist unter https://doi.org/10.1007/s00278-021-00556-9 zufinden. 